\title{
O cuidado na hanseníase e os impactos na formação em Enfermagem: Relato de experiência
}

\author{
The Hansen's disease's care and the impacts on the Nursing graduation: An experience report \\ Los cuidados de la enfermedad de Hansen y los impactos en la graduación de Enfermería: Un \\ informe de experiencia
}

Recebido: 14/05/2021 | Revisado: 20/05/2021 | Aceito: 22/05/2021 | Publicado: 08/06/2021

\author{
Pâmela Ramos Januário \\ ORCID: https://orcid.org/0000-0002-0410-8540 \\ Universidade Federal do Estado do Rio de Janeiro, Brasil \\ E-mail: pamelaramosj@edu.unirio.br \\ Larissa Artimos Ribeiro \\ ORCID: https://orcid.org/0000-0001-7134-5135 \\ Universidade Federal do Estado do Rio de Janeiro, Brasil \\ E-mail: lari.artimos@edu.unirio.br \\ Lilia de Macedo Lanceiro \\ ORCID: https://orcid.org/0000-0002-7491-6287 \\ Universidade Federal do Estado do Rio de Janeiro, Brasil \\ E-mail: lilialanceiro@gmail.com \\ Andressa Teoli Nunciaroni \\ ORCID: https://orcid.org/0000-0001-6469-592X \\ Universidade Federal do Estado do Rio de Janeiro, Brasil \\ E-mail: andressa.nunciaroni@unirio.br \\ Vanessa de Almeida Ferreira Corrêa \\ ORCID: https://orcid.org/0000-0001-7121-4493 \\ Universidade Federal do Estado do Rio de Janeiro, Brasil \\ E-mail: vanessa.correa@unirio.br
}

\begin{abstract}
Resumo
$\mathrm{O}$ artigo relata as práticas de acolhimento a uma pessoa com suspeita de hanseníase e as atividades educativas desenvolvidas com a população durante atividades práticas de graduandos de enfermagem em uma unidade básica de saúde, localizada em um município do estado do Rio de Janeiro (RJ), em outubro de 2019. Frente à situação da hanseníase no Brasil e ao acolhimento de A. refletiu-se acerca das vulnerabilidades sociais e da falta de informação, os quais potencializam o estigma relacionado ao agravo da condição; e redução do acesso ao diagnóstico e tratamento em tempo oportuno. A partir da atividade prática, ampliou-se o conhecimento teórico-prático das discentes sobre o papel do enfermeiro no enfrentamento da hanseníase, enquanto um problema de saúde pública. A atividade de educação em saúde voltada à temática de diagnóstico, tratamento e combate ao estigma desenvolvido como tecnologia leve junto à população, proporcionaram aproximação com usuários e o diálogo quanto à hanseníase. Assim, espera-se incentivar novos projetos de extensão e atividades práticas voltadas à formação de enfermeiros; estudos e sensibilização de discentes, docentes e população quanto ao tema.
\end{abstract}

Palavras-chave: Hanseníase; Vulnerabilidade social; Educação em saúde; Atenção primária à saúde.

\begin{abstract}
The article reports the reception practices to a person with a suspected case of leprosy and the educational activities developed among the community during the practical activities of nursing students in a primary care clinic located in a municipality of the State of Rio de Janeiro (RJ), in october 2019. In view of the leprosy's circumstances in Brazil, and the reception of patient A. it was built a reflection about the vulnerabilities and the lack of information which increase the stigma related to worsening of the condition and decrease the access to diagnostic and timely treatment. In this perspective, from the practical activity, it was experienced the expansion of theoretical and practical knowledge of the students about the nurse's role in facing leprosy as a public health issue. The health education action focused on identifying, treating and fighting against the stigma implemented through the light technology techniques with the population provided approximation and open dialogue regarding leprosy. Therefore, it is expected to encourage new extension projects and practical activities aiming at the nursing development, studies and sensibilization of students, professors as well as the general population regarding the theme.
\end{abstract}

Keywords: Leprosy; Social vulnerability; Health education; Primary health care. 


\begin{abstract}
Resumen
Este artículo relata prácticas de acogida a una persona con lepra y actividades educativas desenvolvidas con la población durante actividades prácticas de estudiantes de grado de Enfermería, realizadas en una unidad básica de salud del municipio de Río de Janeiro en el mes de octubre de 2019. Ante la situación de la lepra en Brasil y la acogida del paciente A, fue posible reflexionar sobre los impactos de las vulnerabilidades sociales y de la falta de información, en la potencialización y propagación del estigma y la reducción del acceso oportuno al tratamiento. En esa perspectiva y a partir de esa actividad práctica, se experimentó la ampliación del conocimiento teórico-práctico de las estudiantes sobre el papel del enfermero en la lucha contra la lepra como problema de salud pública. La acción de la educación en salud dirigida a la temática de la identificación, tratamiento y combate al estigma, proporcionaron aproximación con los usuarios y el diálogo con la comunidad sobre la lepra. De esa forma, se espera incentivar nuevos proyectos de extensión y actividades prácticas de vivencia en la formación de Enfermería, otros estudios y sensibilizar a los estudiantes, a los profesores y a la población sobre el tema.
\end{abstract}

Palabras clave: Lepra; Vulnerabilidad social; Educación en salud; Atención primaria de salud.

\title{
1. Introdução
}

A hanseníase é uma doença crônica, infectocontagiosa, causada pelo Mycobacterium leprae, que afeta principalmente os nervos periféricos e células cutâneas. Essa doença tem evolução lenta e progressiva, porém, é essencial a compreensão de sua capacidade de produzir incapacidades físicas, se não for tratada. A bactéria M. leprae é transmitida pelas vias respiratórias, mas é necessário um contato próximo e prolongado de uma pessoa suscetível com uma pessoa contaminada, que não esteja recebendo o tratamento adequadamente. Sendo assim, as melhores formas de cessar a transmissão são por meio do diagnóstico oportuno e do tratamento precoce (Ministério da Saúde, 2017).

Destacam-se os dados epidemiológicos globais e do Brasil. Segundo a Organização Mundial de Saúde (OMS) foram reportados 208.619 casos novos de hanseníase no mundo em 2018. Desses, 30.957 ocorreram na região das Américas, sendo 28.660 notificados no Brasil. Os respectivos parâmetros classificam o país como de alta carga para a doença (Ministério da Saúde, 2020), sendo, no entanto, a distribuição dos casos de heterogênea, com maior transmissão nas regiões norte, nordeste e centro-oeste (Freitas et al., 2017).

No recorte temporal entre 2014 e 2018 foram diagnosticados, no Brasil, um total de 140.578 casos novos de hanseníase e, dentre esses, 55,2\% ocorreram no sexo masculino, com prevalência na faixa etária de 50 a 59 anos, seguido logo pelo intervalo de 40 a 49 anos de idade (Ministério da Saúde, 2020). Em relação ao ano de 2019, dados preliminares indicam o diagnóstico de 23.612 casos novos de hanseníase no Brasil. O estado do Rio de Janeiro ocupa o $11^{\circ}$ lugar no total de casos novos na população geral (Ministério da Saúde, 2020).

Destaca-se, ainda, que a Hanseníase possui tratamento ambulatorial disponível no Sistema Único de Saúde (SUS), mediado pela equipe de saúde, incluindo os Agentes Comunitários de Saúde (ACS). Os ACS contribuem na comunicação entre a comunidade e os serviços de saúde, uma vez que apresentam maior conhecimento da área adscrita e do cotidiano da população local. Dessa forma, o trabalho dos profissionais em apreço, na atenção à pessoa com hanseníase, está focado na realização de visitas domiciliares ou, quando em situação de rua, visitas aos locais onde a pessoa mais frequentemente se encontra, a fim de supervisionar o tratamento e garantir maior adesão, além de prover atenção às queixas decorrentes de efeitos colaterais ou consequências do desenvolvimento da doença (Sales et al., 2013).

Além do ACS, toda a equipe de saúde se envolve no acompanhamento da pessoa com hanseníase e de seus contatos próximos (Ministério da Saúde, 2017). O enfermeiro é, muitas vezes, o profissional que suspeita da doença, e atuando como um agente de humanização, organiza as intervenções junto aos comunicantes, gerencia o cuidado longitudinal durante o tratamento até a cura e capacita a equipe para a realização das ações de vigilância em saúde (Albano, 2020). Assim, a vivência e discussão sobre o tema é essencial durante a formação profissional do enfermeiro, com intuito de qualificar a vigilância do 
agravo da hanseníase e a atenção às pessoas acometidas, além de potencializar que o acompanhamento à pessoa com hanseníase seja acolhedor, humanizado e eficaz, sem medos ou estigmas por parte dos profissionais (Silva et al., 2016).

No entanto, a ótica de terapêutica têm encontrado correlação significante entre pacientes sob regimes de tratamento irregular, ou seja, já diagnosticados que, no entanto, não seguem as consultas de rotina ou realizam autoadministração dos medicamentos; e situações de vulnerabilidade social, como baixa renda, baixa escolaridade e moradia compartilhada entre muitos familiares. Tais fatos de vulnerabilidade social, são considerados condicionantes para o aumento da transmissão entre os contatos e para a circulação de variações multibacilares da doença (Lopes \& Rangel, 2014).

Assim, tem-se a hanseníase como um grave problema de saúde pública permeado por uma história de preconceitos e de segregação que marca as pessoas e suas famílias acometidas pela doença. Logo, a não valorização do contexto sócio histórico de vida das pessoas acometidas por esta condição pode reproduzir práticas que não levam em consideração os determinantes sociais envolvidos no processo saúde-doença-cuidado. $\mathrm{Na}$ atual diretriz proposta pela OMS para combate à doença como problema de saúde pública (World Health Organization, 2016), as ações para combate à hanseníase são baseadas em três pilares: fortalecer parcerias do governo; promover detecção precoce e tratamento imediato para evitar a incapacidade e a transmissão do bacilo; e barrar a discriminação e promover a inclusão.

Nesta direção, duas reflexões são indispensáveis para compreendermos a complexidade que envolve a atenção à saúde à pessoa com hanseníase. A primeira relacionada ao contexto histórico, o qual não reconhece a determinação social do processo saúde-doença-cuidado das pessoas e a segunda voltada à falta de informações quanto à hanseníase no que se refere ao rompimento dos estigmas envolvidos; à identificação precoce e ao tratamento oportuno; e às estratégias de educação junto à população e de formação em saúde nos cursos de graduação.

O contexto histórico que a hanseníase traz consigo é vasto. É uma doença milenar, com diversos registros desde o quinto século a.c. no sudeste asiático, que afeta homens e mulheres de todas as faixas etárias, mas que pelo potencial de incapacitação físicas, está extremamente associada a um histórico de segregações sócio-espaciais, em "lazaretos" (Opromolla \& Laurenti, 2011). Apesar dessa condição clínica estar diretamente relacionada às questões socioeconômicas, nem sempre o cuidado abrangeu as vulnerabilidades sociais e a atenção à saúde, além da doença. Desde a notificação dos primeiros casos, ainda no século XVII, até o início do século XX, com a criação do Departamento Nacional de Saúde Pública (DNSP) e a Conferência Americana de Lepra, não havia intervenção coordenada visando à mitigação da proliferação dos casos da doença. Esse cenário, associado à exclusividade de cuidados e informações sobre os doentes apenas para médicos filiados ao Departamento de Profilaxia da Lepra (DPL), tornaram difícil o acesso da sociedade às informações acerca da doença (Opromolla \& Laurenti, 2011; Faria \& Santos, 2015).

Com isso, impactos como o preconceito, lacunas nas informações sobre os sintomas e tratamento, falta de diagnóstico em tempo oportuno, pensamentos de que a doença não tem cura ou que as pessoas com hanseníase deveriam viver isoladas, ainda estão enraizados na sociedade. Tais preconceitos dificultam a aceitação do diagnóstico por parte das pessoas acometidas pela doença, prejudicando a testagem de contatos, adesão e continuidade do tratamento (Lopes \& Rangel, 2014; Opromolla \& Laurenti, 2011).

Além disso, vale ressaltar que a discriminação e rejeição são responsáveis por impactos psicológicos e sociais, uma vez que afetam negativamente relações familiares, de trabalho e de amizades; acometendo também a autoestima e trazendo à tona sentimentos de medo, angústia, culpa e exclusão. Tais sentimentos podem, ainda, resultar em abandono ou recidivas da doença, ou mesmo transmissão intrafamiliar (Lopes \& Rangel, 2014).

A fim de se alcançar o cuidado adequado à pessoa com hanseníase se faz necessário partir de dois princípios fundamentais da estrutura de saúde pública: a hierarquização do cuidado, onde a atenção primária colabora com os demais pontos de atenção desde a suspeita e diagnóstico oportuno até o acompanhamento do tratamento e cura; e a equipe 
interdisciplinar, fundamental para a qualidade e efetividade do cuidado, ao considerar as diferentes necessidades em saúde determinadas pelos aspectos sócio-culturais da pessoa, da família e da comunidade.

Apesar do vasto conhecimento acerca da hanseníase e dos muitos desafios que permeiam o processo saúde-doençacuidado na atualidade, a temática ainda é abordada de forma frágil nos Cursos de Graduação na área da saúde (Chaves, 2018). Nesta perspectiva, acredita-se que este relato de experiência pode contribuir para reflexões de discentes, docentes e profissionais no que tange à superação da carga histórica relacionada à hanseníase e à implementação de processos de cuidado que envolvam acolhimento, vigilância, tratamento e cura junto à pessoa com hanseníase, a partir dos determinantes sociais da saúde.

Ainda, espera-se fomentar a importância das atividades práticas na formação em saúde, a partir da vivência na construção de vínculos, relacionamentos interpessoais, identificação das demandas dos usuários e implementação de ações de educação em saúde. Assim, busca-se formar profissionais de saúde com competências teóricas e práticas para construir com a população processos de cuidado em saúde que perpassam a escuta ativa em relação às condições de saúde e capacidade profissional voltada à atuação para a erradicação da hanseníase e de seus estigmas. Assim, os saberes se ampliam para além dos aspectos biológicos da doença, incluindo também as perspectivas emocionais e sociais, os valores de vida, as crenças, as vontades e as subjetividades (Duarte et al. 2009).

Diante do exposto, objetivou-se relatar as práticas de acolhimento à uma pessoa com suspeita de hanseníase e as atividades educativas desenvolvidas com a população durante atividades práticas de graduandos de enfermagem em uma unidade básica de saúde, localizada em um município do estado do Rio de Janeiro (RJ), Brasil (BR).

\section{Metodologia}

Trata-se de estudo qualitativo, do tipo relato de experiência, a partir da vivência prática de acadêmicas de enfermagem. Refletiu-se acerca da determinação social do processo saúde-doença-cuidado das pessoas acometidas pela hanseníase e da formação do enfermeiro voltada ao campo da Atenção Primária em Saúde (APS). A etapa reflexiva foi fundamentada no referencial metodológico da formulação discursiva, pautada na vivência prática das autoras, em conceitos teóricos e referências científicas atuais.

As experiências e ações descritas neste estudo foram realizadas no mês de outubro de 2019, em uma unidade de Atenção Básica localizada em um município do estado do Rio de Janeiro (RJ), Brasil, durante a disciplina de Enfermagem na Atenção Primária à Saúde, ofertada aos alunos do quarto período de um curso de graduação de uma Universidade Federal.

Durante as atividades de ensino prático as discentes tiveram a oportunidade de realizar o acolhimento a uma pessoa com suspeita de hanseníase e atividades de educação em saúde com a população, o que despertou a construção desta reflexão, pautada nos aspectos sociais da atenção às pessoas com hanseníase e nas repercussões para a formação do enfermeiro. Assim, este relato é apresentado em duas seções: a. Momentos de vivência e acolhimento na unidade; e b. Ações de educação em saúde com a população: repercussões no processo de formação do enfermeiro.

\section{Resultados e Discussão}

\subsection{Momentos de vivência e acolhimento na unidade}

A Unidade Básica de Saúde (UBS) onde as discentes vivenciaram o acolhimento à pessoa com suspeita de hanseníase e desenvolveram práticas educativas segue o modelo de atenção da Estratégia de Saúde da Família (ESF). Seu território adscrito conta com significativa parcela de população em situação de rua; famílias com baixa renda e elevada vulnerabilidade social; e áreas de classe média. Para garantir a inserção e seguimento longitudinal da população em situação de rua nos 
serviços do SUS, a unidade conta também com o Consultório na Rua, formado por equipe multiprofissional, que, de forma itinerante, visa a garantir o acesso à atenção integral à saúde, atuando, então, como vínculo entre essa população e a unidade básica (Ministério da Saúde, 2012).

Ainda sobre a população adscrita, nos deparamos com elevada concentração de idosos, dos quais comorbidades, tais como hipertensão arterial sistêmica e diabetes mellitus, são as doenças crônicas mais prevalentes. Apesar disso, pôde-se notar a expressiva presença do público jovem adulto, em idade produtiva, participando dos grupos de gestantes e de combate ao uso de tabaco. A UBS oferece, além dos serviços descritos, a atenção à saúde por meio de ações de: vacinação; consultas de enfermagem e médicas; curativos; procedimentos; farmácia; central de esterilização; consultório na rua; e odontologia. Há também serviços nas áreas de nutrição, psicologia e assistência social, por meio do Núcleo de Apoio à Saúde da Família (NASF).

A vivência das discentes compreende a observação e participação do cotidiano das equipes de saúde da ESF nos diversos setores da unidade, com o objetivo de interagir com a população adscrita, compreender a prática do enfermeiro na ESF e desenvolver competências associadas ao campo da APS. As atividades práticas possibilitam, portanto, oportunidades de aprendizagem ativa com reflexões e ações voltadas à formação em saúde, com atuação nos territórios de vida da população e de trabalho das equipes.

Neste contexto, o contato com o usuário A., do sexo masculino, adulto, ocorreu quando ele procurou a unidade para a realização de um curativo em lesões presentes nas mãos. $\mathrm{O}$ acolhimento ao usuário realizado com base no Processo de Enfermagem (Resolução nº 358, 2009) iniciou-se através da coleta de dados de Enfermagem para que fosse possível identificar seu histórico, já que era um usuário não recorrente da UBS. Durante a anamnese, o usuário se identificou, mencionou que encontrava-se em situação de rua, que estava desempregado devido ao seu estado de saúde, que já havia sido preso e que não tinha mais contato com a família.

Com intuito de obter informações relevantes para formação de um diagnóstico de enfermagem e elaboração de plano de cuidados, realizou-se também o exame físico. Neste momento, identificou-se lesão cutânea em membro inferior esquerdo; mãos em garras, com dedos atrofiados, presença de bolhas com conteúdo seroso e lesões hipocrômicas; e diminuição de sensibilidade dolorosa na pele nos membros superiores. Em relação ao ciclo de convívio, o usuário afirmou já ter tido contato próximo com uma pessoa diagnosticada com hanseníase.

Entende-se que, o diagnóstico da hanseníase pode ser realizado exclusivamente mediante avaliação clínica através da apresentação de um ou mais dos seguintes achados: lesão da pele com diminuição da sensibilidade, acometimento dos nervos com espessamento neural, baciloscopia positiva (Ministério da Saúde, 2016). O paciente A. afirma que realizou múltiplas baciloscopias, em que apenas uma teve o resultado positivo, e por isso ele negava iniciar o tratamento. Esse tipo de comportamento, onde o paciente se recusa a engajar na poliquimioterapia devido a não aceitação da condição de ser portador da doença é um caso infelizmente comum, e esse diagnóstico tardio pode acontecer, entre outros motivos, devido à ausência de informação sobre os sinais e sintomas da hanseníase; à busca tardia de atendimento nos serviços de saúde; e à dificuldade dos profissionais de saúde em identificar a doença (Arantes et al., 2010; Martins \& Donda, 2020).

Durante o diálogo com o usuário A., ocorreu o acolhimento, com postura de escuta ativa; e tanto a conversa quanto o exame físico fluíram de forma leve e com trocas de conhecimentos. Entretanto, a posição de não aceitação do diagnóstico e de resistência ao início do tratamento manifestada pelo usuário trouxe sentimentos de frustração às discentes. A atitude do usuário evidenciou que, nem sempre é possível esclarecer em uma única conversa que o diagnóstico de hanseníase deve ser baseado, essencialmente, no quadro clínico e os resultados dos exames de baciloscopia devem ser correlacionados com a clínica.

Tal percepção trouxe diversas reflexões para as discentes de enfermagem: como dialogar, desde a escolha das palavras mais claras e de fácil compreensão até como explicar a situação diagnóstica e a clínica da doença; das crenças do usuário e 
estigmas sociais que permeiam a aceitação da condição e adesão ao tratamento; das diferentes percepções do risco de complicações e agravos definitivos; da importância do estabelecimento de vínculo na construção de uma relação interpessoal baseada na confiança e diálogo; e da importância da equipe interdisciplinar para o cuidado em saúde.

Realizou-se, então, discussões com a equipe do Consultório da Rua, a fim de construir coletivamente ações em saúde voltadas ao usuário A. para o acompanhamento do seu processo saúde-doença-cuidado, identificação de suas necessidades em saúde, além dos aspectos biológicos da doença e vigilância dos contatos. Notou-se que as Equipes de Saúde da Família e do Consultório na Rua passaram a discutir o caso com vistas a elaborar estratégias de busca ativa e vigilância em saúde para o usuário A.

Assim, a vivência de encontro com o usuário A., a prática de cuidado desenvolvida através do diálogo, do acolhimento, das orientações quanto à hanseníase e de cuidado com a prevenção de incapacidades físicas, associadas à reflexão sobre os determinantes sociais e históricos relacionados à hanseníase e ao usuário A. potencializaram práticas na formação em saúde centradas na pessoa, equipe do Consultório da Rua e de Saúde da Família e as discentes de enfermagem.

Para as pessoas com hanseníase e em situação de elevada vulnerabilidade social, deve-se garantir a atenção em saúde que inclua: prevenção de incapacidades físicas causadas pela doença; educação em saúde para autonomia na tomada de decisões compartilhadas; e capacidade de dialogar sobre sua doença. Essas ações são coordenadas pelas UBS por meio de diversas tecnologias leves (Cecílio, 1997), incluindo a construção de vínculos; ações educativas individuais e coletivas, relacionadas a exercícios preventivos e cuidados específicos com os olhos, mãos e pés; e abordagem interprofissional que inclua os aspectos sociais. Além disso, incluir estratégias voltadas à reabilitação, a fim de diminuir os impactos negativos na rotina diária, colaborando para que haja inserção profissional e social da pessoa (Ministério da Saúde, 2017).

\subsection{Ações de educação em saúde com a população: repercussões no processo de formação do enfermeiro}

Durante a vivência junto ao usuário A., foi possível despertar nas discentes a importância de se conhecer as dificuldades, os medos e o grau de desamparo experimentado pela pessoa com hanseníase e, ainda, articular tais reconhecimentos à família/ pessoas do convívio próximo e à comunidade adscrita.

Ao refletir sobre o caso do usuário A. e a necessidade do desenvolvimento de tecnologias leves articuladas com os princípios do SUS, tais como a equidade, integralidade e universalidade (Lei $\mathrm{n}^{\circ}$ 8080, 1990), as discentes perceberam a importância de se expandir a reflexão e o diálogo acerca da hanseníase junto à população, por meio de estratégias de educação em saúde. A educação em saúde é definida pelo Ministério da Saúde (2012) como "Processo educativo de construção de conhecimentos em saúde que visa à apropriação temática pela população [...] para aumentar a autonomia das pessoas no seu cuidado". Com base nessa definição e mediante as informações obtidas no caso do usuário A., o grupo de discentes e docente idealizaram, em parceria com a equipe de saúde, uma forma de discutir com a população os aspectos sociais, históricos e de saúde, referentes à hanseníase. Dessa forma, planejou-se uma ação com o objetivo de dialogar sobre os sinais e sintomas de hanseníase, promover o diagnóstico precoce e prevenir o agravo da doença.

Criou-se, então, o "Varal de informações", uma tecnologia de educação em saúde desenvolvida pelas discentes na sala de espera da UBS. Esta ação foi um jogo dinâmico, composto por oito ilustrações elaboradas pelas discentes, com temas selecionados a partir das Diretrizes do Ministério da Saúde (2017; 2016): auto exame, lesões hipocrômicas, alterações da sensibilidade, forma de transmissão, testes diagnósticos, tratamento medicamentoso e acompanhamento do tratamento. Algumas imagens representavam informação verdadeira e outras falsa, essa identificação era realizada em conjunto, entre discentes e usuários, presentes na sala de espera, seguida de diálogo sobre o tema.

Ao final, a população que participou era encorajada a selecionar os pontos mais importantes apreendidos com a ação educativa. Observou-se, por meio da experiência vivenciada, que ações de educação em saúde, quando realizadas de forma 
dialogada e inclusiva, apresentam a potência de criar espaços onde é possível esclarecer dúvidas, trocar conhecimentos e romper o estigma social da hanseníase. Além disso, percebeu-se que ações como esta, com discussões de forma lúdica e sem julgamentos, demonstram elevado poder em formar multiplicadores das informações.

Para potencializar esse efeito de multiplicação, foram distribuídos panfletos informativos elaborados pelas discentes, contendo as ilustrações verdadeiras trabalhadas na tecnologia "Varal de Informações" e breves orientações baseadas nas Diretrizes de enfrentamento à hanseníase recomendadas pelo Ministério da Saúde (2016) sobre a prevenção, controle e identificação precoce da hanseníase. O panfleto apoia a expansão da informação, uma vez que os usuários poderiam levá-los para suas casas, locais de trabalhos e divulgar intencionalmente o conhecimento adquirido, sendo expandido o diálogo da experiência vivenciada com os familiares e amigos.

Além disso, ações de educação em saúde voltadas para o diálogo e intercâmbio de saberes possuem potência para ampliar o grau de conhecimento acerca da hanseníase. Estudo de Moreira et al. (2014) aponta que intervenção educativa realizada junto à população geral em uma UBS contribui para o aumento do conhecimento sobre as formas de transmissão; os sinais, sintomas e complicações; e o tratamento da doença.

Destaca-se que a ação educativa desenvolvida pelas discentes teve grande receptividade pela população e pela equipe de saúde; além do interesse por parte da população quanto ao tema, negligenciado nos dias atuais. Tanto na dinâmica realizada quanto na distribuição de panfletos, os usuários se mostraram sempre atentos ao diálogo construído junto aos discentes e participaram ativamente do momento, dando continuidade à conversa entre si, por meio do compartilhamento de experiências pessoais e conhecimentos prévios. Acredita-se que ações de educação em saúde, quando implementadas de forma dialogada e que atendem às necessidades regionais ou territoriais, aumentam as chances de promover mudanças de comportamentos efetivas, seja com relação ao autocuidado para a prevenção primária; ou redução de agravos ou controle e cura de condições clínicas (Ministério da Saúde, 2013).

Por isso, a atividade de ensino-prático, além de proporcionar às discentes de enfermagem o primeiro contato com a UBS, ampliou a abordagem do cuidado para além do aspecto biológico da doença, valorizando, principalmente, o contexto social, econômico e territorial no qual o usuário está inserido; e possibilitou o diagnóstico oportuno de uma pessoa com hanseníase, pois o usuário foi também acolhido pela equipe de saúde. Ainda, a vivência possibilitou perceber a importância do trabalho da equipe do Consultório na Rua e da ESF, além de planejar, implementar e incentivar os projetos de educação em saúde. Dessa forma, a experiência foi muito além do que cumprir um componente curricular, visto que as discentes desenvolveram a compreensão de como os determinantes e as vulnerabilidades sociais, tais como: renda; moradia; escolaridade; emprego; crenças; e questões psicológicas e comportamentais impactam na qualidade de vida e na saúde das pessoas. Assim, foi possível compreender, a partir do encontro e das reflexões do presente estudo, os determinantes sociais da saúde e construir formas de diálogos e ações de educação em saúde para a ampliação do acesso à informação; além da sensibilização quanto à importância do enfrentamento da hanseníase.

Tendo como um dos objetivos para a formação do enfermeiro a vivência dos princípios do SUS na prática, a disciplina configurou-se de grande importância para as discentes na construção do conhecimento por meio da experiência da escuta, do acolhimento e do vínculo, visto que as atividades de ensino-prático evidenciaram a necessidade de proporcionar à população e aos grupos em situação de vulnerabilidade a atenção à saúde, multiprofissional e integral.

Além disso, no âmbito da hanseníase, as discentes vivenciaram a importância do cuidado integral e acolhedor, no qual é necessário expandir a visão simplista de saúde somente como cura de doenças; e pensar nos determinantes sociais e nas vulnerabilidades em saúde; nas práticas de autocuidado e na adesão ao tratamento; vigilância de novos casos e de agravos; e combate ao estigma ao construir junto à população o acesso à informação em saúde. No âmbito da sociedade, as atividades realizadas contribuíram para o engajamento da população e sua participação em assuntos relacionados à saúde e qualidade de 
vida, alinhadas às Diretrizes da OMS de combate à hanseníase como problema de saúde pública (WHO, 2016). Abordagens voltadas para a construção de diálogos e trocas de experiências, independentemente do tema inicial proposto, abrem caminho para discussão de diferentes linhas de cuidado e colocam em evidência situações envolvidas no processo saúde-doença-cuidado que poderiam estar negligenciadas.

\section{Considerações Finais}

A vivência discente, expressa neste relato de experiência contribui, portanto, para o incentivo à formação voltada para a promoção da saúde e vigilância da hanseníase e seus agravos. Apenas através do encontro com os usuários e profissionais nos diferentes locais de cuidado em saúde na APS é possível construir estratégias para melhorar o acesso, diagnóstico precoce e oportuno; a qualidade de vida dos usuários e erradicar o estigma relacionado à hanseníase. Evidenciou-se assim, a necessidade de projetos e atividades extensionistas sobre promoção da saúde e desenvolvimento de ações de educação em saúde; e prevenção e vigilância do agravo da hanseníase, com intuito de aumentar a vivência de discentes com a comunidade e formar profissionais com perfis capazes de agir em diferentes contextos sociais.

Neste sentido, identificou-se a importância da realização de atividades de ensino-prático no campo da APS, através de disciplinas curriculares obrigatórias, para a vivência real de encontro e cuidado com a população; além da sensibilização de discentes quanto ao enfrentamento necessário à hanseníase, no momento da formação em saúde. Atenta-se também para a escassez de estudos sobre a hanseníase e a prática de cuidado do enfermeiro na APS.

O presente relato de experiência contribui para apontar a essencialidade da vivência prática no processo de formação em enfermagem, por meio de disciplinas obrigatórias, ligas acadêmicas e projetos de extensão universitária. Sugere-se o desenvolvimento de novos estudos que possam contribuir para a qualidade do cuidado do enfermeiro no campo da APS e produção de evidências científicas, associadas à discussão da hanseníase na formação em saúde, visto a necessidade de seu enfrentamento enquanto um problema de saúde pública.

\section{Agradecimentos}

Ao paciente A. que tanto nos ensinou. Aos profissionais de saúde que compartilharam conosco seus saberes e práticas em saúde.

\section{Referências}

Albano, M. L., Sousa, A. A. S. de, Roscoche, K. G. C., Oliveira Filho, P. R. V. de, \& Felício, J. F. (2020). Barriers to the integrality of care for people with hanseniasis: perception of Nurses. Research, Society and Development, 9(8), e531985864. https://doi.org/10.33448/rsd-v9i8.5864

Arantes, C. K, Garcia, M. L. R., Filipe, M. S., Nardi, S. M. T. \& Paschoal, V. D. (2010). Avaliação dos serviços de saúde em relação ao diagnóstico precoce da hanseníase. Epidemiologia e Serviços de Saúde, 19(2), 155-164. http://dx.doi.org/10.5123/S1679-49742010000200008

Cecílio, L. C. O. (1997). Uma sistematização e discussão de tecnologia leve de planejamento estratégico aplicada ao setor governamental. In: Merhy EE; Onocko R (Org.). Agir em saúde: um desafio para o público (pp.151-167). Hucitec Editora.

Chaves, A. E. P. (2018). O ensino da atenção à hanseníase em cursos de graduação em enfermagem [Tese de Doutorado, Universidade Federal do Rio Grande do Norte].

Duarte, M. T. C., Ayres, J. A. \& Simonetti, J. P. (2009). Consulta de enfermagem: estratégia de cuidado ao portador de hanseníase em atenção primária. Rev. Texto \& Contexto - Enfermagem, 18 (1), 100-7 https://doi.org/10.1590/S0104-07072009000100012

Faria, L. \& Santos, L. A. de C. (2015) A hanseníase e sua história no Brasil: a história de um "flagelo nacional". História, Ciências, Saúde-Manguinhos, 22(4), 1491-5. https://doi.org/10.1590/S0104-59702015000400016

Freitas, L. R. S., Duarte, E. C. \& Garcia, L. P. (2017) Análise da situação epidemiológica da hanseníase em uma área endêmica no Brasil: distribuição espacial dos períodos 2001 - 2003 e 2010 - 2012. Revista Brasileira de Epidemiologia, 20 (4), 702-13. https://doi.org/10.1590/1980-5497201700040012

Lei $n^{\circ}$ 8080, Lei Orgânica de Saúde 1990 (DOU) (Brasil). http://www.planalto.gov.br/ccivil_03/Leis/L8080.htm 
Research, Society and Development, v. 10, n. 6, e52210616099, 2021

(CC BY 4.0) | ISSN 2525-3409 | DOI: http://dx.doi.org/10.33448/rsd-v10i6.16099

Lopes, V. A. S. \& Rangel, E. M. (2014) Hanseníase e vulnerabilidade social: uma análise do perfil socioeconômico de usuários em tratamento irregular. Saúde em Debate, 38 (103), 817-29. https://doi.org/10.5935/0103-1104.20140074

Martins, W. S. \& Donda, P. (2020) Incapacidades da hanseníase: causas do diagnóstico tardio. Revista Corpus Hippocraticum, 1(1). http://revistas.unilago.edu.br/index.php/revista-medicina/article/view/238

Ministério da Saúde. (2012). Glossário Temático: Gestão do trabalho e da educação na saúde. Secretaria de Gestão do Trabalho e da Educação na Saúde. http://bvsms.saude.gov.br/bvs/publicacoes/glossario_gestao_trabalho_2ed.pdf

Ministério da Saúde. (2012). Manual Sobre o Cuidado à Saúde Junto à População em Situação de Rua. Secretaria de Atenção à Saúde. 189.28.128.100/dab/docs/publicacoes/geral/manual_cuidado_populalcao_rua.pdf

Ministério da Saúde. (2013). Diretrizes para o cuidado das pessoas com doenças crônicas nas redes de atenção à saúde e nas linhas de cuidado prioritárias. Secretaria de Atenção à Saúde $\quad$ Departamento de Atenção https://bvsms.saude.gov.br/bvs/publicacoes/diretrizes\%20_cuidado_pessoas\%20_doencas_cronicas.pdf

Ministério da Saúde. (2016). Diretrizes para vigilância, atenção e eliminação da hanseníase como problema de saúde pública. Secretaria de Vigilância em Saúde. https://portalarquivos2.saude.gov.br/images/pdf/2016/fevereiro/04/diretrizes-eliminacao-hanseniase-4fev16-web.pdf

Ministério da Saúde. (2017). Guia prático sobre a hanseníase. Secretaria de Vigilância em Saúde. portalarquivos2.saude.gov.br/images/pdf/2017/novembro/22/Guia-Pratico-de-Hanseniase-WEB.pdf

Ministério da Saúde. (2020). Boletim Epidemiológico Hanseníase. Secretaria de Vigilância em Saúde, Brasil. http://www.aids.gov.br/pt-br/pub/2020/boletimepidemiologico-de-hanseniase-2020

Moreira, A. J., Naves, J. M., Fernandes, L. F. R. M., Castro, S. S. de \& Walsh, I. A. P. de. (2014) Ação educativa sobre hanseníase na população usuária das unidades básicas de saúde de Uberaba-MG. Saúde em Debate, 38(101), 234-43. https://doi.org/10.5935/0103-1104.20140021

Opromolla, P. A., \& Laurenti, R. (2011) Controle da hanseníase no Estado de São Paulo: análise histórica. Revista de Saúde Pública, 45 (1), $195-203$. https://www.scielosp.org/article/rsp/2011.v45n1/195-203/pt/\#ModalArticles.

World Health Organization. (2016). Global Leprosy Strategy 2016-2020: Accelerating towards a leprosy-free world. Regional Office for South-East Asia of World Health Organization. https://apps.who.int/iris/handle/10665/208824

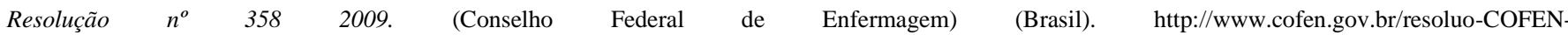
3582009_4384.html\#: :text=RESOLU\%C3\%87\%C3\%83O\%20COFEN-

358\%2F2009.\%20Disp\%C3\%B5e\%20sobre\%20a\%20Sistematiza\%C3\%A7\%C3\%A3o\%20da\%20Assist\%C3\%AAncia,cuidado\%20profissional\%20de\%20E nfermagem $\% 2 \mathrm{C} \% 20 \mathrm{e} \% 20 \mathrm{~d} \% \mathrm{C} 3 \% \mathrm{~A} 1 \% 20$ outras $\% 20$ provid $\% \mathrm{C} 3 \%$ AAncias

Sales, J. C. S., Luz, V. L. E. S., Branco, F. M. F. C., Araújo, M. F. F. de, Castro, S. H. T. de S. \& Silva, T. C. A. da. (2013). O significado da hanseníase para o agente comunitário de saúde. Revista Interdisciplinar, 6(1), 17-24. https://revistainterdisciplinar.uninovafapi.edu.br/index.php/revinter/article/view/5/pdf_5

Silva, L. S. R. da, Silva, T. M. da, Rocha, J. T., Andrade, W. J. de, Lessa, E. C. \& Correia, N. da S. (2016). A assistência de enfermagem aos portadores de hanseníase assistidos pelo programa de saúde da família. Revista de Enfermagem da Universidade Federal de Pernambuco - UFPE (online), 10 (11), $4111-7$. https://doi.org/10.5205/1981-8963-v10i11a11498p4111-4117-2016 